\title{
Analysis of the Process of Energy Transformation in Magnetohydrodynamic Stirrer of Liquid Metals with Nonsinusoidal Current
}

\author{
Viktor N. Timofeev ${ }^{1,2}$, Eduard R. Vinter ${ }^{1,2}$, Maksim Y. Khatsayuk ${ }^{1,2}$, \\ Aleksandr V. Fomin ${ }^{1,2}$, Natalia S. Shakhoval ${ }^{1,2}$. \\ ${ }^{1}$ Siberian Federal University, Krasnoyarsk, Russia \\ 2 "RPC Magnetic Hydrodynamics", Krasnoyarsk, Russia
}

\begin{abstract}
This article describes the electromagnetic field analytical model of magnetohydrodynamic (MHD) stirrer with nonsinusoidal current, built with the account of the longitudinal edge effect. The solution is obtained in the form of a Fourier series in a comprehensive way. Differential and integral properties of the system have been defined. There have been received differential and integral characteristics, which are the basis for building the power supply source and control systems for the liquid metal stirring process.
\end{abstract}

Index Terms - Magnetohydrodynamics, liquid metals stirring, analytical calculation, nonsinusoidal periodic voltage, Fourier series transformation.

\section{INTRODUCTION}

$\mathrm{I}$ $\mathrm{N}$ RECENT YEARS there have increased production and consumption of aluminum-based alloys. The technology of multicomponent alloys preparation implements an important operation, which is the homogenization of chemical composition and temperature of the melt (liquid metal) in the full volume of the alloying furnace bath [1]. The use of MHD stirrers enables to automate the homogenization process, to reduce the preparation time and decrease the power consumed for the production of high-quality alloys [2-5].

Normally, the power of MHD stirrer is supplied through the low frequency sinusoidal voltage [2, 3]. However, currently, the development of power conversion equipment raises interest to the use of non-sinusoidal periodic voltage as power source for MHD stirrer. The non-sinusoidal voltage can take the form of a single-pole rectangular pulses, alternating rectangular pulses, triangular pulses or a sequence of pulses of a sinusoidal voltage.

Currently, a number of studies have been completed on the effectiveness of using the non-sinusoidal pulse electromagnetic fields in the units designed for liquid metal stirring. In $[6,7]$ there have been carried out numerical and experimental studies on the heat transfer of conductive liquid in low frequency pulse periodic electromagnetic field. In addition, a number of works are dedicated to the numerical $[8,9]$ and experimental $[10,11]$ study into ingot solidification process as exposed to the pulse periodic magnetic field.

These works showed a specific advantage of using nonsinusoidal periodic fields in the units designed for the electromagnetic stirring of melt. However, no theoretical study of the energy conversion process and the nature of electromagnetic fields distribution has been performed.

This paper presents the analytical solution to the problem of the electromagnetic field distribution in MHD stirrer with nonsinusoidal periodic current, taking into account the longitudinal edge effect and the discrete distribution of linear current load. There have been identified differential and integral characteristics, which are the basis for building the power supply source and control systems.

\section{PROBLEM STATEMENT}

The sketch of the holding furnace with MHD stirrer installed under the bath bottom is presented in Fig. 1.

Fig. 1 displays: 1 is alloying furnace bath; 2 is liquid metal (melt); 3 is electric heaters; 4 is polyphase winding of the inductor; 5 is inductor core;

The length of the core $L=2 \rho \tau_{\text {ind }}$, where $\rho$ is the number of poles pairs; $\tau_{\text {ind }}$ is a polar pitch.

When the multiphase ( $m$-number of phases) winding is connected to the source of periodic sinusoidal or nonsinusoidal voltage the inductor generates a travelling magnetic field. The speed of movement of the magnetic induction amplitude in the direction of the axis $x$ is equal to $u_{1}=2 \tau f$, where $f$ is the current frequency in the winding of the inductor. Exposed to the travelling magnetic field, the melt starts to move with speed $u$ also in the direction of the axis $x$ performing the stirring of melt.

Having adopted similar [4] assumptions, we obtain the design model of the MHD stirrer presented in Fig. 2.

Linear current density in the current sheet is defined as follows

$$
j_{m}(t)=\frac{W_{m} \cdot i_{m}(t)}{2 \Delta_{m}},
$$


where $i_{m}(t) ; W_{m}$ is the instantaneous current and a turning number in $m$ th seam.

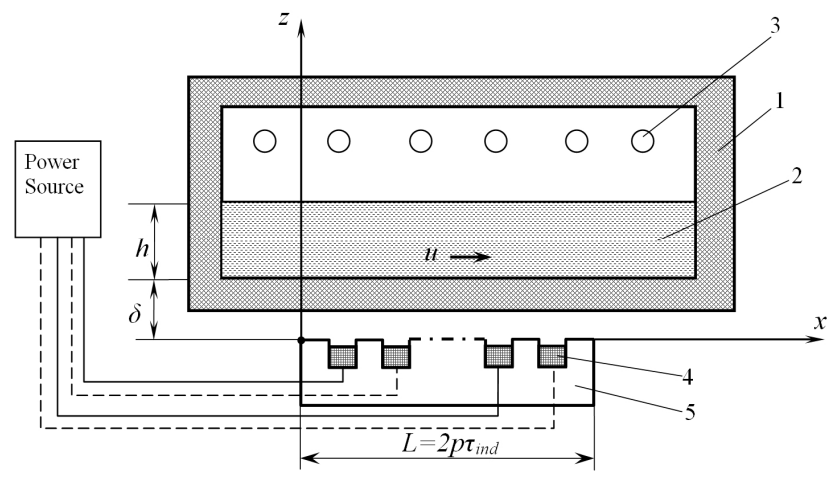

Fig. 1. Sketch - alloying furnace with MHD stirrer

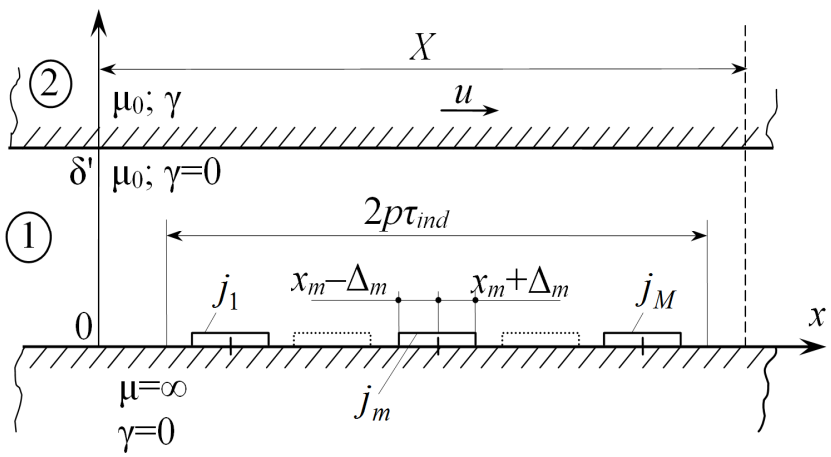

Fig. 2. Design model of MHD stirrer

To obtain a travelling magnetic field in the inductor it is required to install in the inductor two or more windings that are supplied from the source with the same shape, frequency and voltages, phase-shifted relative to each other. Suppose that the inductor has $M$ seams and linear current load of each subsequent phase is phase-shifted relative to linear current load of the previous seam by the angle $T / M$. Then, for the $m$ th seam the linear current load will be determined in the range of

where

$$
t_{m}-\frac{T}{2} \prec t \prec t_{m}+\frac{T}{2},
$$

$$
t_{m}=\frac{T}{2}+\frac{T}{M}(m-1) .
$$

Thus, linear current density in the $\mathrm{m}$ th seam is defined as follows

$$
j_{m}(t)=\left\{\begin{array}{cc}
A-2 A e^{-\frac{\left(t-\left(t_{m}-\frac{T}{2}\right)\right)}{\tau}} ; t_{m}-\frac{T}{2} \prec t \prec t_{m} \\
-A+2 A e^{-\frac{\left(t-t_{m}\right)}{\tau} ;} \quad t_{m} \prec t \prec t_{m}+\frac{T}{2}
\end{array}\right.
$$

Here

$$
A=J-2 J e^{-\frac{T}{2 \tau}}
$$

$$
\begin{aligned}
& \tau=L / R ; \\
& J=\frac{U}{R} \cdot \frac{W}{2 \Delta} ;
\end{aligned}
$$

where $J$ is an amplitude of linear current density, $A / m ; T$ is the period, $s ; L$ is the inductivity, $H ; R$ is the copper resistance, $\Omega ; U$ is the voltage amplitude, $V ; W$ is the number of turns in the seam; $2 \Delta$ is the width of current sheet, $m$.

Fig. 3 displays graphs of voltage and linear current density at various $\tau$.

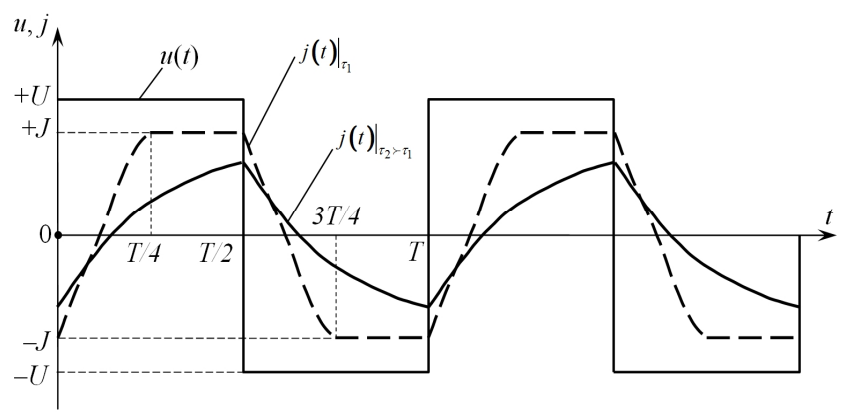

Fig. 3. Graphs of instantaneous value of voltage and current linear density

\section{THEORY}

\section{A. Electromagnetic field equations and boundary condition}

This model of electric- and magnetic -fields vector have the following elements:

$$
\bar{E}(z, x, t)=\bar{e}_{y} E_{y} ; \bar{H}_{1,2}(z, x, t)=\bar{e}_{x} H_{x 1,2}+\bar{e}_{z} H_{z 1,2} \text {. }
$$

Electric-field vector $E_{y}$ satisfies the functions of equations [4] (index " $y$ " is omitted):

in area $1,0 \leq z \leq \delta^{\prime}$

$$
\frac{\partial^{2} E_{1}}{\partial z^{2}}+\frac{\partial^{2} E_{1}}{\partial x^{2}}=0
$$

in area 2

$$
\frac{\partial^{2} E_{1}}{\partial z^{2}}+\frac{\partial^{2} E_{1}}{\partial x^{2}}-\mu_{0} \gamma \frac{\partial E_{2}}{\partial t}-\mu_{0} \gamma u \frac{\partial^{2} E_{2}}{\partial x}=0
$$

where $\gamma$ is specific electrical conductivity of melt, $\Omega^{-1} \cdot m^{-1} ; u$ is the speed of melt movement, $\mathrm{m} / \mathrm{s}$; $\mu_{0}=4 \pi \cdot 10^{-7} \mathrm{H} / \mathrm{m} ; t$ is time, $s$.

Below are the area boundary conditions that are found true:

$$
\begin{gathered}
\frac{\partial E_{1}}{\partial z}(0, x, t)=\left\{\begin{array}{l}
\mu_{0} \frac{\partial j_{m}}{\partial t}, x_{m}-\Delta_{m} \prec x \prec x_{m}+\Delta_{m} \\
0
\end{array}\right. \\
E_{1}\left(\delta^{\prime}, x, t\right)=E_{2}\left(\delta^{\prime}, x, t\right) \\
\frac{\partial E_{1}}{\partial z}\left(\delta^{\prime}, x, t\right)=\frac{\partial E_{2}}{\partial z}\left(\delta^{\prime}, x, t\right) \\
E_{2}(\infty, x, t)=0
\end{gathered}
$$




\section{B. Solving electromagnetic field equations}

The required functions $E_{1,2}(z, x, t)$ are periodical in time $t$ with the period $T$, thus, the solutions shall be searched as Fourier series of a complex form [12]

$$
E_{1,2}(z, x, t)=\sum_{k=-\infty}^{k=+\infty} E_{1,2 k}(z, x) e^{i \zeta_{k} t}
$$

where

$$
\begin{gathered}
E_{1,2 k}(z, x)=\frac{1}{T} \int_{0}^{T} E_{1,2}(z, x, t) e^{-i \zeta_{k} t} \partial t ; \\
\zeta_{k}=\frac{2 k \pi}{T} .
\end{gathered}
$$

We convert the differential equations (1), (2) and the boundary conditions (3) to (6) in compliance with (7) and (8). We multiply these expressions by function $\frac{1}{T} e^{-i \zeta_{k} t}$ and integrate the resulting expressions by $t$ from 0 to $T$, in the result we obtain

$$
\begin{gathered}
\frac{\partial^{2} E_{1 k}}{\partial z^{2}}+\frac{\partial^{2} E_{1 k}}{\partial x^{2}}=0 ; \\
\frac{\partial^{2} E_{2 k}}{\partial z^{2}}+\frac{\partial^{2} E_{2 k}}{\partial x^{2}}-i \zeta_{k} \mu_{0} \gamma E_{2 k}-\mu_{0} \gamma u \frac{\partial E_{2}}{\partial x}=0 ; \\
\frac{\partial E_{1 k}}{\partial z}(0, x)= \begin{cases}\psi_{k m}, & x_{m}-\Delta_{m} \prec x \prec x_{m}+\Delta_{m} \\
0\end{cases} \\
E_{1 k}\left(\delta^{\prime}, x\right)=E_{2 k}\left(\delta^{\prime}, x\right) ; \\
\frac{\partial E_{1 k}}{\partial z}\left(\delta^{\prime}, x\right)=\frac{\partial E_{2 k}}{\partial z}\left(\delta^{\prime}, x\right) ; \\
E_{2 k}(\infty, x)=0 .
\end{gathered}
$$

Here

$$
\psi_{k m}=i \mu_{0} \zeta_{k} J \sum_{m=1}^{M}\left(I_{k m 1}+I_{k m 2}\right)
$$

where

$$
\begin{aligned}
& I_{k m 1}=i \frac{A}{2 k \pi}\left(e^{-i \zeta_{k} t_{m}}-e^{-i \zeta_{k}\left(t_{m}-\frac{T}{2}\right)}\right)+ \\
& +\frac{2 A e^{\frac{t_{m}-T / 2}{\tau}}}{\left(\frac{T}{\tau}+i 2 k \pi\right)}\left(e^{-t_{m}\left(\frac{1}{\tau}+i \xi_{k}\right)}-e^{-\left(t_{m}-\frac{T}{2}\right)\left(\frac{1}{\tau}+i \xi_{k}\right)}\right)
\end{aligned}
$$

$$
\begin{aligned}
& I_{k m 2}=-\left[i \frac{A}{2 k \pi}\left(e^{-i \zeta_{k}\left(t_{m}+\frac{T}{2}\right)}-e^{-i \xi_{k} t_{m}}\right)+\right. \\
& \left.+\frac{2 A e^{\frac{t_{m}}{\tau}}}{\left(\frac{T}{\tau}+i 2 k \pi\right)}\left(e^{-\left(\frac{1}{\tau}+i \zeta_{k}\right)\left(t_{m}+\frac{T}{2}\right)}-e^{-t_{m}\left(\frac{1}{\tau}+i \xi_{k}\right)}\right)\right]
\end{aligned}
$$

Assuming that the required functions are periodical also in the coordinate $x$ with the period $X$, we can have the following

$$
E_{1,2 k}(z, x)=\sum_{n=-\infty}^{n=+\infty} E_{1,2 k n}(z) e^{i \xi_{n} x}
$$

where

$$
\begin{gathered}
E_{1,2 k n}=\frac{1}{X} \int_{0}^{X} E_{1,2 k n}(z, x) e^{-i \xi_{n} x} \partial x ; \\
\xi_{n}=\frac{2 n \pi}{X} .
\end{gathered}
$$

Similarly, we convert equations (9), (10) and the boundary conditions (11) to (14) in compliance with (15) and (16), in the result we obtain

$$
\begin{gathered}
\frac{d^{2} E_{1 k n}}{d z^{2}}-\xi_{n}^{2} E_{1 k n}=0 ; \\
\frac{d^{2} E_{2 k n}}{d z^{2}}-\phi_{k n}^{2} E_{2 k n}=0 ; \\
\frac{d E_{1 k n}}{d z}(0)=\psi_{k n} ; \\
E_{2 k n}(\infty)=0 ; \\
E_{1 k n}\left(\delta^{\prime}\right)=E_{2 k n}\left(\delta^{\prime}\right) ; \\
\frac{d E_{1 k n}}{d z}\left(\delta^{\prime}\right)=\frac{d E_{2 k n}}{d z}\left(\delta^{\prime}\right) ;
\end{gathered}
$$

where

$$
\begin{gathered}
\phi_{k n}^{2}=\xi_{n}^{2}+i \zeta_{k} \mu_{0} \gamma+i \mu_{0} \gamma u \xi_{n} ; \\
\psi_{k n}=i \frac{2 \mu_{0} \zeta_{k} J}{X \xi_{n}} N_{k n} ; \\
N_{k n}=\sum_{m=1}^{M}\left(\hat{I}_{k m 1}+\hat{I}_{k m 2}\right) \sin \left(\xi_{n} \Delta_{m}\right) e^{-i \xi_{n} x_{m}} ;
\end{gathered}
$$

Common solutions are as follows

$$
\begin{gathered}
E_{1 k n}=C_{1} e^{\xi_{n} z}+C_{2} e^{-\xi_{n} z} ; \\
E_{2 k n}=C_{3} e^{\phi_{k n} z}+C_{4} e^{-\phi_{k n} z} .
\end{gathered}
$$

Having defined constant integrations $C_{1} \div C_{4}$ from the boundary conditions (19) to (22) and having input them in (23) and (24), we make some simple transformations and obtain 
Here

$$
\begin{aligned}
& E_{1 k n}(z)=-\frac{\psi_{k n}}{\xi_{n}} \frac{G_{k n}(z)}{Q_{k n}} \\
& E_{2 k n}(z)=-\psi_{k n} \frac{e^{\phi_{k n}(\delta-z)}}{Q_{k n}} .
\end{aligned}
$$

$$
\begin{gathered}
G_{k n}(z)=\xi_{n} \operatorname{ch} \xi_{n}\left(z-\delta^{\prime}\right)-\phi_{k n} \operatorname{sh} \xi_{n}\left(z-\delta^{\prime}\right) \\
Q_{k n}=\xi_{n} \operatorname{sh} \xi_{n} \delta^{\prime}+\phi_{k n} \operatorname{ch} \xi_{n} \delta^{\prime}
\end{gathered}
$$

Given (7) and (15), we apply Euler formula, we obtain the following for area 1

$$
\begin{aligned}
& E_{1}(z, x, t)=-\frac{\mu_{o} X J}{\pi T} \sum_{k=-\infty}^{\infty} \sum_{n=-\infty}^{\infty} \frac{k}{n^{2}} D_{k n}(z) \times \\
& \times \cos \left(\zeta_{k} t+\xi_{n} x+\beta_{k n}\right)
\end{aligned}
$$

Here

$$
D_{k n}=\left|i \frac{G_{k n}(z)}{Q_{k n}} N_{k n}\right| ; \beta_{k n}=\arg \left(i \frac{G_{k n}(z)}{Q_{k n}} N_{k n}\right) .
$$

\section{Differential and integral properties}

Maxwell equation allows to define the vertical component of magnetic induction

$$
\begin{aligned}
& B_{z 1}(z, x, t)=\int \frac{\partial E_{1}(z, x, t)}{\partial x} \partial t= \\
& =-\frac{\mu_{o} J}{\pi} \sum_{k=-\infty}^{\infty} \sum_{n=-\infty}^{\infty} \frac{1}{n} D_{k n}(z) \cos \left(\zeta_{k} t+\xi_{n} x+\beta_{k n}\right)
\end{aligned}
$$

Instantaneous electromagnetic (EM) power generated in the inductor winding is equal to

$$
\begin{aligned}
& P(t)=-l \sum_{m=1}^{M} \int_{x_{m}-\Delta_{m}}^{x_{m}+\Delta_{m}} E_{1}(0, x, t) j_{m}(t) \partial x= \\
& =\frac{\mu_{0} X^{2} l J^{2}}{\pi^{2} T} \sum_{k=-\infty}^{\infty} k \sum_{n=-\infty}^{\infty} \frac{1}{n^{3}} D_{k n}(0) C_{k n}(t)
\end{aligned}
$$

where

$$
\begin{aligned}
& C_{k n}(t)=\sum_{m=1}^{M} \hat{j}(t) \int_{x_{m}-\Delta_{m}}^{x_{m}+\Delta_{m}} \cos \left(\zeta_{k} t+\xi_{n} x+\beta_{k n}\right) \partial x= \\
& =\sum_{m=1}^{M} \hat{j}_{m}(t) \sin \left(\xi_{n} \Delta_{m}\right) \cos \left(\zeta_{k} t+\alpha_{k n m}\right)
\end{aligned}
$$

where

$$
\hat{j}(t)=j(t) / J ; \alpha_{k n m}=\xi_{n} x_{m}+\beta_{k n} .
$$

Instantaneous EM force acting on the winding of the inductor is defined by expression

$$
\begin{aligned}
& f(t)=-l \sum_{m=1}^{M} \int_{x_{m}-\Delta_{m}}^{x_{m}+\Delta_{m}} B_{z 1}(0, x, t) j_{m}(t) \partial x= \\
& =\frac{\mu_{0} l X J^{2}}{\pi^{2}} \sum_{k=-\infty}^{\infty} \sum_{n=-\infty}^{\infty} \frac{1}{n^{2}} D_{k n}(0) C_{k n}(t)
\end{aligned}
$$

We find the average values for the period of EM force and power in the relative units, taking the following as basic values

$$
P_{b}=\frac{4 \mu_{0} \tau^{2} l J^{2}}{T} ; F_{b}=\frac{P_{b}}{2 \tau} T .
$$

The average EM power and force for the period are

$$
\begin{aligned}
& \hat{P}_{e m}=\sum_{k=-\infty}^{\infty} \sum_{n=-\infty}^{\infty} \frac{k}{n^{3}} \hat{D}_{k n}(0) K_{k n} ; \\
& \hat{F}_{e m}=\sum_{k=1}^{\infty} \sum_{n=1}^{\infty} \frac{1}{n^{2}} \hat{D}_{k n}(0) K_{k n} .
\end{aligned}
$$

Here

$$
\begin{gathered}
K_{k n}=\sum_{m=1}^{M} \sin \left(\xi_{n} \Delta_{m}\right)\left(V_{k n m}^{(1)}+V_{k n m}^{(2)}\right) ; \\
V_{k n m}^{(1)}=\frac{1}{T} \int_{t_{m}-\frac{T}{2}}^{t_{m}} \hat{j}(t) \cos \left(\zeta_{k} t+\alpha_{k n m}\right) \partial t ; \\
\hat{G}_{k n}^{(2)}(z)=\frac{1}{T} \int_{t_{m}}^{t_{m}+\frac{T}{2}} \hat{j}(t) \cos \left(\zeta_{k} t+\alpha_{k n m}\right) \partial t ; \\
c h\left[\frac{2 n}{\hat{X}}\left(z-\delta^{\prime}\right)\right]-\hat{\varphi}_{k n} s h\left[\frac{2 n}{\hat{X}}\left(z-\delta^{\prime}\right)\right] \\
\hat{Q}_{k n}=\frac{2 n}{\hat{X}} s h\left(\frac{2 n}{\hat{X}} \delta^{\prime}\right)+\hat{\varphi}_{k n} c h\left(\frac{2 n}{\hat{X}} \delta^{\prime}\right) ; \\
\varepsilon_{1}=\frac{\gamma \mu_{0} \zeta_{k=1} \tau^{2}}{\pi^{2}}=\frac{2 \gamma \mu_{0} \tau^{2}}{\pi T} ; s_{1}=1-\frac{u}{2 \tau} T ; \\
\hat{\varphi}_{k n}=\sqrt{\left(\frac{2 n}{\hat{X}}\right)^{2}+i \varepsilon_{1}+i \varepsilon_{1} \frac{2 n}{\hat{X}}\left(1-s_{1}\right) ; \hat{X}=X / \tau .}
\end{gathered}
$$

\section{DISCUSSION OF RESULTS}

Fig. 4 shows the graph of instantaneous value distribution for the relative magnetic induction density within the slot $\left(B_{z 1} / \mu_{0} J\right)$. Tooth and slot ripples emerge on the inductor surface, however, their manifestation on the melt surface is reducing. In addition, the non-sinusoidal supply voltage affects the dynamics of distribution for the travelling magnetic field but does not affect the nature of its distribution in the gap. 
Fig. 5 shows the graphs of instantaneous EM force for the period when the melt velocity is zero. It can be seen that the force changes during the period, in contrast to a two-phase machine with a symmetrical sinusoidal current, when the instantaneous force does not change versus time [13]. Moreover, the nature of the change in force is not sinusoidal and is characterized by moments of sharp decline and increase in the amplitude. Such a character of EM force change makes it possible to generate the impulse mechanical effect in the melt, which allows to perform stirring and homogenization at the level of microinhomogeneities.

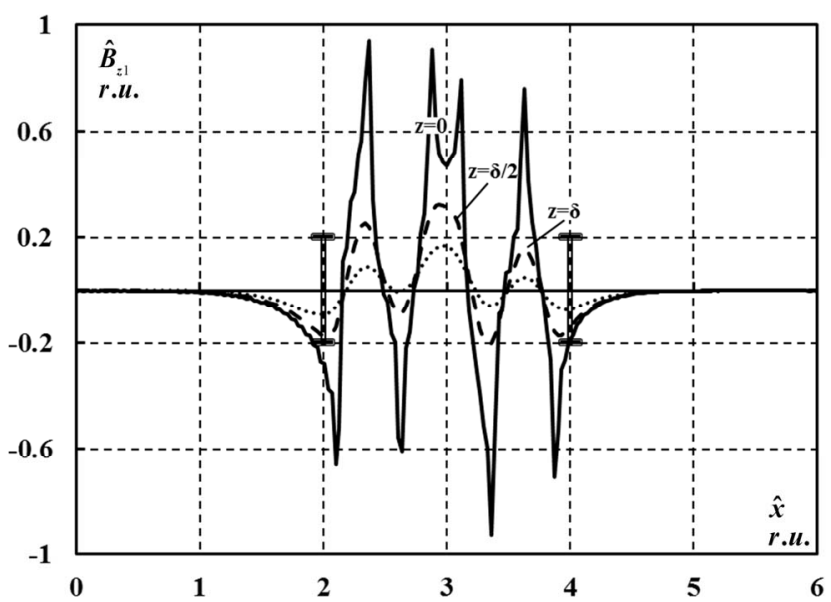

Fig. 4. Graph of distribution for the instantaneous relative magnetic induction in the gap taking into account the longitudinal edge effect for $t=0, \tau=0.05 T, s=1, \varepsilon=3.6, X=6 \tau_{\text {ind }}$

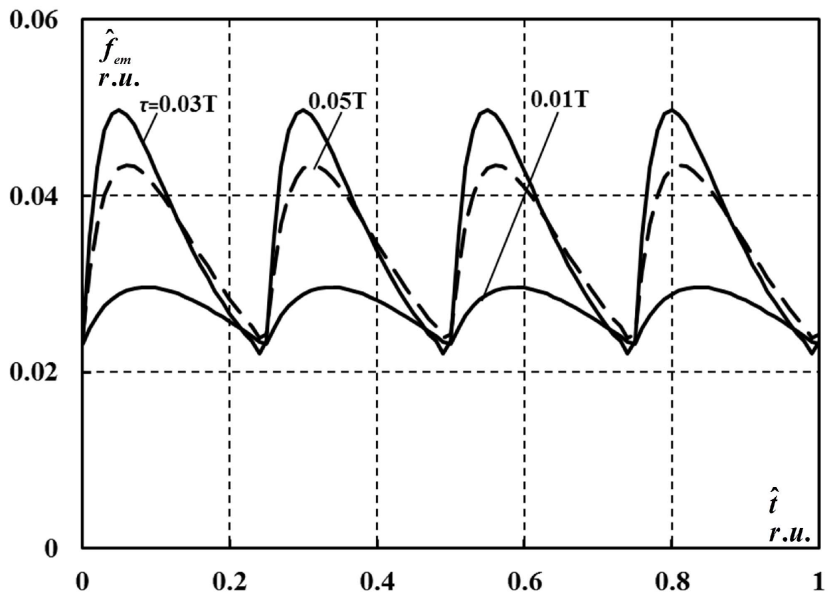

Fig. 5. Graph of instantaneous relative EM force during the period taking into account the longitudinal edge effect $\tau=0.05 T, s=1, \varepsilon=3.6, X=6 \tau_{\text {ind }}$

Fig. 6 and 7 give the relations of the average values for the period of EM power and force obtained from sliding at different values of $\mathrm{Q}$ factor $\varepsilon$. As can be seen from Fig. 6, 7 , the non-sinusoidal nature of the supply voltage has a minor impact on the energy and mechanical characteristics, while the curves shape is close in nature to the case of MHD stirrer with a classic sinusoidal power supply [4].

\section{FINDINGS AND CONCLUSIONS}

1. The $2 \mathrm{D}$ design model has been built to analyze the electromagnetic field and electromagnetic characteristics of MHD stirrer of liquid metal with non sinusoidal current.

2. The application of double integral conversions using Fourier series with complex coefficients enabled to obtain analytical expressions for electromagnetic fields vectors and electromagnetic characteristics of MHD stirrer.

3. The analysis has been completed on the magnetic induction distribution in the device gap, instantaneous EM power and force for the period non sinusoidal current. The results obtained can be used to eliminate inhomogeneities in the process of multi-component aluminum alloys production.

4. The obtained integral characteristics for power and force can be used to develop a power source and stirring process control systems.

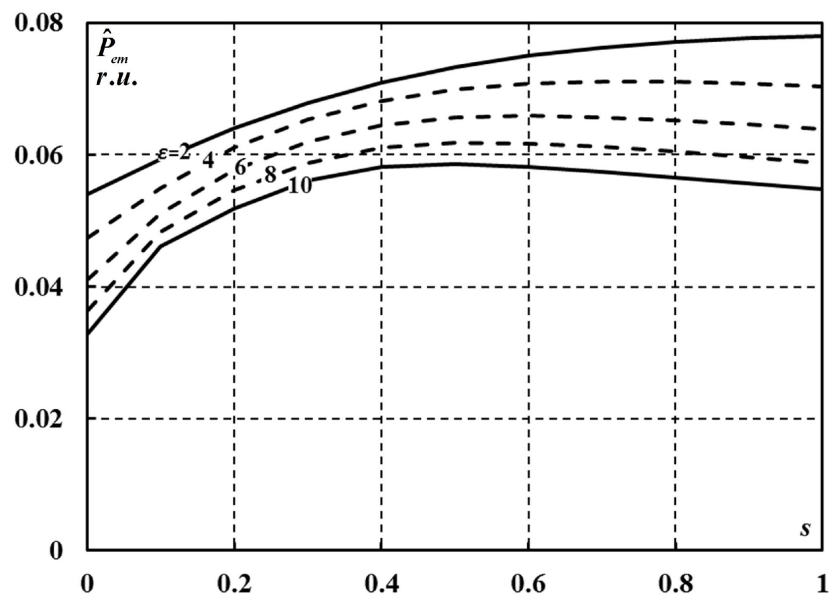

Fig. 6. Relation of relative EM power from sliding average for the period taking into account the longitudinal edge effect for $\tau=0.05 T, X=6 \tau_{\text {ind }}$

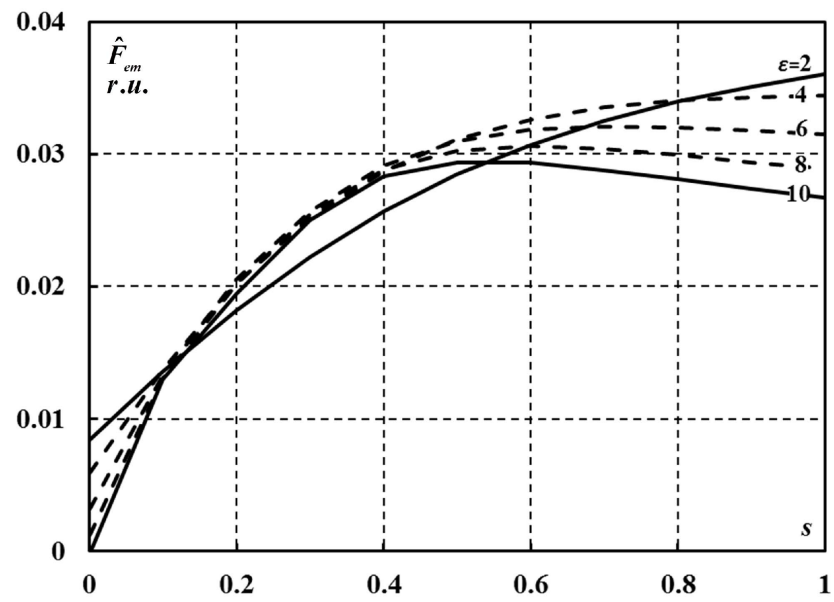

Fig. 7. Relation of relative EM force from sliding average for the period taking into account the longitudinal edge effect for $\tau=0.05 T, X=6 \tau_{\text {ind }}$ 


\section{ACKNOWLEDGEMENTS}

This research has been completed with the financial support of the Russian Fund of Fundamental Research and the Government of Krasnoyarsk Territory within the framework of Research Project No. 18-48-242013 "Research into the impact of spatial and time-andfrequency distributions of electromagnetic forces in the melt on the nature of the MHD processes in liquid metal".

\section{REFERENCES}

[1] Nacke B. Theoretical Background and Aspects of Electrotechnologies: Physical Principles and Realization: Intensive Course. Basic I. Publishing house of ETU; 2012.

[2] Fdhila RB, Sand U, Eriksson JE, et al. A stirring history. ABB Review. 2016;3:45-48.

[3] Timofeev VN, Khatsayuk MY. Theoretical Design Fundamentals for Mhd Stirrers for Molten Metals. Magnetohydrodynamics. 2016 OctDec;52(4):495-506. PubMed PMID: WOS:000394414000006; English.

[4] Timofeev VN, Khatsyuk MY. Analytical calculation of differential and integral electromagnetic characteristics of mhd-stirrer. Journal of Siberian Federal University Engineering \& Technologies. 2017;10(1):74

[5] K. Bolotin, I. Smolyanov, E. Shvydkiy [et al.] // Numerical simulation of the electromagnetic stirrer adapted by using magnetodielectric composite. Magnetohydrodynamics. - 2017. - V. 53. - № 4. - P. 723-730.

[6] Musaeva D, Ilin V, Baake E, et al. Numerical Simulation of the Melt Flow in an Induction Crucible Furnace Driven by a Lorentz Force Pulsed at Low Frequency. Magnetohydrodynamics. 2015 OctDec;51(4):771-783. PubMed PMID: WOS:000369117200010; English.

[7] Musaeva D, Baake E, Ilin V, et al. Analysis of the Almgsi-Alloy Structure Formed under the Influence of Low-Frequency Pulsed Lorentz Force. Magnetohydrodynamics. 2017 Apr-Jun; 53(2):245254. PubMed PMID: WOS:000406079700003; English.

[8] Eckert S, Nikrityuk PA, Rabiger D, et al. Efficient melt stirring using pulse sequences of a rotating magnetic field: Part I. Flow field in a liquid metal column. Metall Mater Trans B. 2007 Dec;38(6):977-988. doi: $\quad 10.1007 / \mathrm{s} 11663-007-9096-4 . \quad$ PubMed PMID: WOS:000251925700015; English.

[9] Guo-Jun, Chen, Zhang Yong-Jie, and Yang Yuan-Sheng. "Modelling the unsteady melt flow under a pulsed magnetic field." Chinese Physics B 22.12 (2013): 124703.

[10] Willers B, Eckert S, Nikrityuk PA, et al. Efficient melt stirring using pulse sequences of a rotating magnetic field: Part II. Application to solidification of Al-Si alloys. Metall Mater Trans B. 2008 Apr;39(2):304-316. doi: 10.1007/s11663-008-9126-x. PubMed PMID: WOS:000255879400014; English.

[11] Gao Y-L, Li Q-S, Gong Y-Y, et al. Comparative study on structural transformation of low-melting pure $\mathrm{Al}$ and high-melting stainless steel under external pulsed magnetic field. Materials Letters. 2007;61(18):4011-4014.

[12] Zill D, Wright WS, Cullen MR. Advanced engineering mathematics. Jones \& Bartlett Learning; 2011.

[13]Neyman, L. Demirchan. K. "Three-phase circuit calculation" in Theoretical backgrounds of electrical engineering, Vol. 1. Leningrad, Leningrad, Energoizdat, 1981. -536 p.

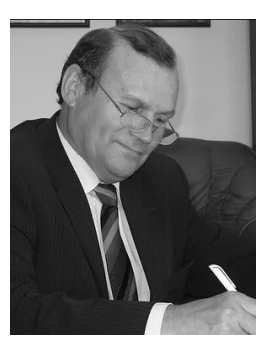

Viktor N. Timofeev was born in 1950, in the Ermolayevo Village, Balakhta District, Krasnoyarsk Territory. In 1974, he completed his education in Krasnoyarsk Polytechnical Institute majoring in Power Plants. In 1978, he was awarded the degree of the Candidate of Technical Sciences. In 1994, he was awarded the degree of the Doctor of Technical Sciences.

Area of expertise: electromagnetic fields analysis; research into electrical process equipment and MHD technology for metallurgical purposes; linear electric machines; methods of complex mathematical and physical simulation; computer aided design of electrical process equipment.

He is the member of the Thesis Board for awarding titles of Candidate and Doctoral degree, the author of over 200 scientific publications, including 2 monographs, 8 educational books, and 70 inventor's certificates and patents. Since 1990, he has been heading the "Research and Production Center of Magnetic Hydrodynamics", Ltd ("RPC Magnetic Hydrodynamics" Ltd), which designs and supplies electrical process equipment and technology to smelters.

$\mathrm{He}$ is Doctor of Technical Sciences, Professor, Academician in Academy of Electrotechnical Sciences, Head of the Department of Electrical Engineering and Electric Process Equipment in the Polytechnical. In 2002, he was awarded the diploma of the winner of the all-Russian competition "Engineer of the year" in the nomination "Electrical equipment". In 2006, he was awarded a label pin of "Honorary worker of science and technology of the Russian Federation".

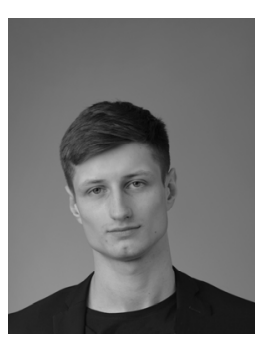

Eduard R. Vinter was born in Severomuysk, Republic of Buryatia, in 1995. In 2016, he received a Master's Degree in Electrical Engineering in Metallurgy at the Siberian Federal University (Krasnoyarsk). Since 2016, he is laboratory assistant at the Power Electronics Department at "RPC Magnetic Hydrodynamics" Ltd. Since 2018, he is assistant of the Electrical Equipment and Eletrical Process Technology Department in the Polytechnic Institute of the Siberian Federal University. Area of expertise: study of electromagnetic, thermal and hydrodynamic processes in magnetohydrodynamic installations of metallurgical purpose. Participant of international scientific and technical exhibitions and conferences. He is the author of 6 publications.

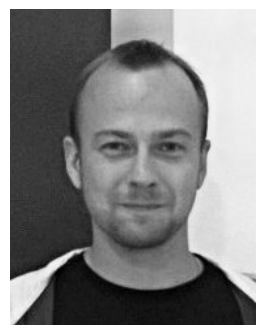

Maksim Y. Khatsayuk was born in 1988 in Minusinsk Town, Krasnoyarsk Territory. He was qualified as an Engineer in specialty of "Electric process units and systems" in the Siberian Federal University (Krasnoyarsk) in 2010, and was awarded the degree of Candidate of Technical Sciences specializing in "Electric machines" in the Ural Federal University (Yekaterinburg) in 2014.

In 2014 - 2018, he held the position of Teaching Assistant, Senior Lecturer, Associate Professor of the Electrical Equipment and Electrical Process Technology Department in the Polytechnic Institute of the Siberian Federal University. In 2010 - 2018, he is an Engineer, Researching Engineer, Head of the Mathematical Simulation Department at "RPC Magnetic Hydrodynamics" Ltd. He is the author of 4 methodological and educational books, 65 publications, 12 patents in the field of electrical process technology and MHD-equipment for metallurgy, acted as a Manager and Executor of 14 R\&D projects. Area of expertise: research into related electromagnetic, thermal, magnetic and hydrodynamic processes in metallurgical units with a view to obtain materials of new physical-mechanical properties and enhanced safety, environment and energy efficiency of the electrical process equipment. 


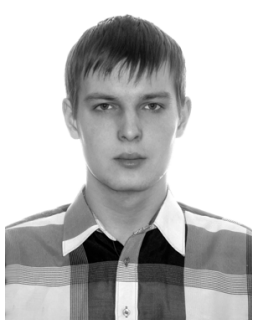

Alexander V. Fomin was born in Krasnoyarsk in 1993. In 2016, he received a Master's Degree in in Electrotechnics in Metallurgy in the Siberian Federal University (Krasnoyarsk). Since 2015, he has been employed in the position of Senior Assistant with Physical Modeling Department, "RPC Magnetic Hydrodynamics" Ltd. Since 2016, he has taken the postgraduate course at the Siberian Federal University. Since 2018, he is the Teaching Assistant of the Electrical Equipment and Eletrical Process Technology Department in the Polytechnic Institute of the Siberian Federal University. Area of expertise: study of electromagnetic, thermal and hydrodynamic processes in MHD units of metallurgy application.

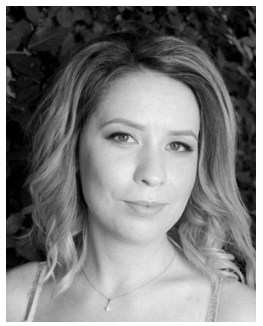

Natalia S. Shakhoval was born in the Solenoozernoye Village, Krasnoyarsk Territory. In 2015, she received Master's Degree, Siberian federal University. She has presently completed the third year of post-graduation studies. Starting from 2016, she is the Teaching Assistant at the department of Electric Technologies in Metallurgy, Siberian federal University. She is the author of 15 articles. Area of expertise: MHD technology in metallurgy, innovations in the field of magnetic hydrodynamics, electromagnetic fields analysis. 